\title{
Giant Pulses from PSR B0540-69 in the Large Magellanic Cloud
}

\author{
Simon Johnston ${ }^{1}$ and Roger W. Romani ${ }^{2}$
}

\begin{abstract}
We report the discovery of the first giant pulses from an extragalactic radio pulsar. Observations of PSR B0540-69 in the Large Magellanic Cloud made with the Parkes radio telescope at $1.38 \mathrm{GHz}$ show single pulses with energy more than 5000 times that of the average pulse energy. This is only the second young pulsar, after the Crab, to show giant pulse emission. Similar to the Crab pulsar, the giant pulses occur in two distinct phase ranges and have significant arrival time jitter within these ranges. The location of the giant pulses appears to lag the peak of the (sinusoidal) X-ray profile by 0.37 and 0.64 phase, although absolute timing between the radio and $\mathrm{X}$-ray data is not yet secure. The dispersion measure of the giant pulses is $146.5 \mathrm{~cm}^{-3} \mathrm{pc}$, in agreement with the detection of the pulsar at $0.64 \mathrm{GHz}$ by Manchester et al. (1993). The giant pulses are scatter broadened at $1.4 \mathrm{GHz}$ with an exponential scattering time of $0.4 \mathrm{~ms}$ and have an emission bandwidth of at least $256 \mathrm{MHz}$. In $8 \mathrm{hr}$ of integration we have failed to detect any integrated flux density from the pulsar to a level of $13 \mu \mathrm{Jy}$, assuming a duty cycle of $10 \%$. This implies the spectral index between 0.64 and $1.38 \mathrm{GHz}$ is steeper than -4.4 .
\end{abstract}

Subject headings: neutron stars: individual: PSR B0540-69

\section{Introduction}

PSR B0540-69 was discovered in the early 1980s by Seward, Harnden \& Helfand (1984) using data from the Einstein X-ray Observatory. The pulsar is located inside the supernova remnant SNR 0540-693 in the Large Magellanic Cloud. It has a short rotation period ( $\sim 50 \mathrm{~ms}$ ) and a rapid spindown with a characteristic age of only $1500 \mathrm{yr}$. There are strong similarities between this system and the Crab. The pulsars have similar characteristics and the plerion around PSR B0540-69 is very similar to the Crab Nebula in size and

\footnotetext{
${ }^{1}$ School of Physics, University of Sydney, NSW 2006, Australia. email simonj@physics.usyd.edu.au

${ }^{2}$ Dept. of Physics, Stanford University, Stanford, CA 94305-4060, USA. email rwr@astro.stanford.edu
} 
energetics (Manchester, Staveley-Smith \& Kesteven 1993). Following the discovery of the X-ray pulsations from PSR B0540-69, optical pulsations were soon detected (Middleditch \& Pennypacker 1985), but the radio pulsations remained elusive. Finally, Manchester et al. (1993; hereafter M93) discovered a broad, weak radio pulse at $0.64 \mathrm{GHz}$. The flux density at that frequency is $0.4 \mathrm{mJy}$ and the pulse duty cycle is more than $80 \%$ with the hint of a double profile. This is similar to the high energy profiles (de Plaa, Kuiper \& Hermsen 2003) which also appear to show a broad, unresolved double structure. M93 failed to detect the pulsar at either 1.4 or $0.44 \mathrm{GHz}$.

In comparison, the radio profile of the Crab pulsar is complex. It consists of a so-called pre-cursor component and main and interpulse components. The pre-cursor has a very steep spectral index rendering it undetectable above about $1 \mathrm{GHz}$. The main and interpulse emission are in phase with the X-ray and $\gamma$-ray emission which both have narrow peaks. At frequencies above $5 \mathrm{GHz}$, further components have been detected (Moffett \& Hankins 1999). The Crab pulsar also produces giant pulses when the emission can exceed the mean pulse energy by a factor of 1000 or more. This giant pulse emission occurs at the phases of the main pulse and interpulse but not at those of the pre-cursor or the 'anomalous' high frequency components (Lundgren et al. 1995; Hankins et al. 2003).

Giant pulses have now also been discovered from the millisecond pulsars PSRs B1937+21 (Cognard et al. 1996) and B1821-24 (Romani \& Johnston 2001). Cognard et al. (1996) noticed that when pulsars are ordered by their magnetic field strength at the light cylinder, the pulsars with giant pulses are highly ranked. Romani \& Johnston (2001) noted the

similarity between the phases of the giant pulse emission and the X-ray emission in these pulsars and speculated that the giant pulse emission may also originate from near the light cylinder. McLaughlin \& Cordes (2003) have very recently reported the non-detection of giant pulses from PSR B0540-69 through observations made at 0.66 and $1.52 \mathrm{GHz}$.

\section{Observations and Data Reduction}

Observations were made on 20 May 2001 (MJD 52049), with the Parkes 64-m radio telescope. We used the center beam of the 21-cm multi-beam system at an observing frequency of $1.38 \mathrm{GHz}$. The receiver had a system equivalent flux density of $27 \mathrm{Jy}$ on cold sky. The back-end consisted of a filterbank system containing 512 channels per polarization, each of width $0.5 \mathrm{MHz}$ for a total bandwidth of $256 \mathrm{MHz}$. The polarization pairs were summed to form the total power, each output was then sampled at $80 \mu \mathrm{s}$, one-bit digitized, and written to DLT for off-line analysis. A total of $8 \mathrm{hr}$ of integration on PSR B0540-69 was obtained, split into 4 individual observations, each of duration $2 \mathrm{hr}$. 
To search for giant pulses, it is not necessary to know the rotational frequency of the pulsar but it is necessary to know the dispersion measure (DM). As an initial trial, we used the DM of $146 \mathrm{~cm}^{-3} \mathrm{pc}$ given in M93. After applying the appropriate delay to each frequency channel, the 512 channels were summed. The mean and rms of groups of 8192 samples (0.65 s) were examined and those which showed obvious signs of interference were discarded. Our nominal $5 \sigma$ sensitivity in $80 \mu \mathrm{s}$ is $0.9 \mathrm{Jy}$. In practice, even after the removal of high sigma points (clipping), we experienced substantially larger background fluctuations and the rms exceeded our expected rms by a factor of $\sim 2$. The entire $8 \mathrm{hr}$ observation $\left(3.6 \times 10^{8}\right.$ samples $)$ was then searched for peaks. This technique was successfully used to discover giant pulses in PSR B1821-24 (Romani \& Johnston 2001) and the giant micro-pulses in PSR B1706-44 (Johnston \& Romani 2002). Two large amplitude, resolved pulses were discovered. We were able to determine the DM of these single pulses by subdividing the total bandwidth into 16 and fitting a straight line to their time-of-arrival across the 16 sub-bands. The DM of both pulses was determined to be $146.5 \pm 0.2 \mathrm{~cm}^{-3}$ pc. Armed with this knowledge we then applied a DM of $146.5 \mathrm{~cm}^{-3} \mathrm{pc}$ to the entire data set.

We then used a more sophisticated search technique to detect weaker giant pulses. The DM introduces a broadening of $220 \mu$ s across each $0.5 \mathrm{MHz}$ channel and the full width at half maximum of the strong giant pulses was $\sim 600 \mu \mathrm{s}$, or 8 samples. We therefore first smoothed the data using a top hat function with a width of 8 samples and then searched the data looking for 4 consecutive data points more than $3 \sigma$ from the mean.

\section{Pulse Timing and Phase Comparisons}

In order to obtain the correct pulsar phase for each time sample, an accurate timing solution for the pulsar is required. The latest published ephemeris for PSR B0540-69 from X-ray timing with the Rossi X-ray Timing Explorer (RXTE) does not cover the epoch of our observations (Zhang et al. 2001) and we therefore used an updated ephemeris provided to us by F. Marshall and W. Zhang from RXTE observations near MJD 52050, including overlap with our observing epochs. The peak of the X-ray profile at the solar system barycenter occurred $0.0366 \mathrm{~s}$ after MJD 52050 and at this epoch the spin frequency was $19.7886114394 \mathrm{~s}^{-1}$ and the frequency derivative was $-1.875580 \times 10^{-10} \mathrm{~s}^{-2}$. The position of the pulsar used in the ephemeris was that obtained from optical observations by Caraveo et al. (1992). Sample RXTE arrival times on the same dates as the radio observations agree with the adopted ephemeris, with uncertainties $\lesssim 0.5 \mathrm{~ms}$. Using this timing solution we were therefore able to form an integrated profile of the entire $8 \mathrm{hr}$ observation, and we assert that uncertainties in the X-ray ephemeris would be insufficient to broaden the pulse by more than $\sim 0.5 \mathrm{~ms}(\sim 1 \%$ 
of the pulse period).

Direct comparison of the phases of the radio giant pulses with the X-ray profile is, however, subject to some uncertainty. We believe the absolute timing in the radio to be correct, as we have obtained arrival times for both the Crab pulsar and PSR B1937+21 which agree with independent radio ephemerides. However, the absolute X-ray timing is not secure and it is known that there are problems comparing RXTE timing of PSR B0540-69 and those made with the Chandra X-ray Observatory (A. Rots, private communication).

\section{Integrated Profile}

We failed to detect any significant integrated pulsed emission from the pulsar. The bottom panel of Fig. 1 shows the pulse profile with 128 phase bins after 8 hr of observations. The $1-\sigma \mathrm{rms}$ is $80 \mu \mathrm{Jy}$. As a check against the possibility that the X-ray ephemeris was incorrect we also performed a Fast Fourier Transform on each de-dispersed $2 \mathrm{hr}$ dataset independently. No significant signals were detected near the expected pulsar frequency. Assuming the pulsar has a duty cycle of $\sim 10 \%$, the upper limit on the continuum flux density at $1.38 \mathrm{GHz}$ is therefore only $13 \mu \mathrm{Jy}$ compared to $400 \mu \mathrm{Jy}$ at $0.64 \mathrm{GHz}(\mathrm{M} 93)$. This implies a spectral index steeper than -4.4 between the two frequencies, although this could be relaxed if the duty cycle of the profile is large and/or the $0.64 \mathrm{GHz}$ flux density is incorrect. This is extremely high, even by pulsar standards, but it is comparable with the spectral index of the pre-cursor component in the Crab pulsar. The distance to the Large Magellanic Cloud is $49.4 \mathrm{kpc}$, hence the luminosity of the pulsar at $1.38 \mathrm{GHz}$ is less than $32 \mathrm{mJy} \mathrm{kpc}{ }^{2}$. Although this is low compared to the luminosity of other young pulsars, and a factor of 100 less than the luminosity of the Crab pulsar, it is significantly higher than that of the recently discovered PSR J0205+6449 in the SNR 3C58 (Camilo et al. 2002).

\section{Giant Pulses}

As described above, the initial search of the data detected two giant pulses. These are shown in the top panel of Fig. 1 where both the entire profile is shown and a more detailed view of the structure in the giant pulses. The widths of these pulses are entirely consistent with an intrinisic delta function convolved with the sampling rate $(80 \mu \mathrm{s})$, the dispersion delay $(220 \mu \mathrm{s})$ and an exponential tail with a $1 / \mathrm{e}$ time of $0.4 \mathrm{~ms}$. This exponential broadening is likely the result of interstellar scattering. The two pulses are offset in phase by 0.03 .

Using the analysis described in Section 2 (data smoothing plus searching for several 
large consecutive samples), we searched a range of DMs in order to determine the cut-off above which no spurious signals were detected. The best results were obtained using the criteria that four consecutive samples should exceed $3 \sigma$. We applied this analysis on the data at a DM of $146.5 \mathrm{~cm}^{-3} \mathrm{pc}$ and detected 15 giant pulses down to this sensitivity limit. Fig. 2 shows the flux density as a function of phase for the 15 detected giants superposed on the X-ray profile taken from De Plaa et al. (2003). There are two separate phase regions for the giant pulses, the first between phases 0.36 and 0.45 and the second between 0.61 and 0.66 phase. These phases should be considered preliminary in light of the discussion about absolute timing above. This is similar to the Crab giant pulses which also occur in two separate phase regions. However, the jitter in the phases of the giants in PSR B0540-69 is much larger than in the Crab; $\sim 5 \mathrm{~ms}$ compared to $\sim 0.5 \mathrm{~ms}$. Fig. 3 shows the histogram of the flux densities obtained. Although we are dealing only with small number statistics, it seems clear that the slope is not as steep as the slope of -3.5 seen in the Crab pulsar (Lundgren et al. 1995) or that of -2.8 seen in PSR B1937+21 (Kinkhabwala \& Thorsett 2000).

Two other points are worth noting. The first is that the brightest giant obtained has a flux density which exceeds the mean single pulse flux density by a factor $\gtrsim 5000$. This is a larger factor than any giant pulse seen in the Crab pulsar. All the giants detected here exceed the mean flux density by 700. Expressed in these terms, this rate of giants (1 in 35000) exceeds that of the Crab pulsar (1 in 350000; see Lundgren et al. 1995) by an order of magnitude. However, in terms of the emitted energy, the giants in PSR B0540-69 are significantly less powerful than in the Crab pulsar.

Finally, the non-detection of giant pulses in PSR B0540-69 at $1.5 \mathrm{GHz}$ by McLaughlin \& Cordes (2003) can probably be attributed to their observational setup. Their sampling rate was $0.6 \mathrm{~ms}$, however, the dispersion smearing across each of their $5 \mathrm{MHz}$ filters was $\sim 2 \mathrm{~ms}$ and this is sufficient to dilute the giant pulse enough for it to fall below their quoted detection threshold of $0.4 \mathrm{Jy}$. Their non-detection of giant pulses at $0.66 \mathrm{GHz}$ would imply that either the spectral index of the giant pulses are shallower than -3 , in contrast to the integrated profile, or that there is no giant pulse emission at this frequency. The latter seems unlikely given that both the Crab and PSR B1937+21 show giant pulse emission at frequencies well below $0.66 \mathrm{GHz}$. McLaughlin \& Cordes (2003) do not comment on a detection or non-detection of the integrated pulse at this frequency. 


\section{Discussion}

PSR B0540-69 is the 4th pulsar known to emit giant pulses and there are now two young pulsars and two millisecond pulsars which emit giants. We detected the giant pulses without detecting the underlying integrated pulsed emission. The four pulsars are in the top five pulsars when ranked by magnetic field at the light cylinder; only PSR B1957+20 from this list does not show giant emission. Clearly therefore this parameter is important to our understanding of the giant pulse phenomenon. Furthermore, all four objects have pulsed, non-thermal, X-ray emission. In the Crab, PSRs B1937+21 and B1821-24 the Xray emission appears to be in phase with the giant radio emission. It is intriguing that for PSR B0540-69, the two giant pulse windows are $\sim 0.5$ out of phase with the X-ray pulse and that their spacing is similar to that of the high energy pulse components. Whether this indicates an error in the relative phasing between the radio and X-ray obserations or an association of the giant pulses with the magnetic pole opposite the high energy emission remains to be tested with independent phase comparisons.

Given the exponential broadening of the giant pulses at $1.38 \mathrm{GHz}$, it seems likely that the integrated profile at $0.64 \mathrm{GHz}$ is dominated by scattering (M93). Scatter broadening scales with the inverse fourth power of the frequency and hence should be $\sim 20$ times larger at $0.64 \mathrm{GHz}$ than at $1.38 \mathrm{GHz}$. The $0.64 \mathrm{GHz}$ profile is thus consistent with a double peaked profile separated by $\sim 0.3$ phase and broadened by $\sim 10 \mathrm{~ms}$ of scattering. It is tempting to relate the location of this double peaked structure with the location of the giant pulses detected at the higher frequency. Further observations at $0.64 \mathrm{GHz}$ are needed to confirm this. Furthermore, the scattering at high frequency would also explain the non-detection of the pulsar at $0.44 \mathrm{GHz}$ reported by M93, where the scattering time would exceed the pulse period. These values also indicate that the effects of scintillation are not important in the giant pulse search toward PSR B0540-69, contrary to the suggestion of McLaughlin \& Cordes (2003).

The DM is high compared to the other known pulsars in the Large Magellanic Cloud which have DMs in the range $67-107 \mathrm{~cm}^{-3} \mathrm{pc}$ (McConnell et al. 1991). A scattering time of $\sim 0.4 \mathrm{~ms}$ at $1.38 \mathrm{GHz}$ is also large, especially when the scattering screen is likely to be located much closer to the pulsar (i.e. in the LMC) than to the Earth. The situation is analogous to that seen in the Vela pulsar, where both the DM and the scattering are much larger than expected for its distance. Both the excess DM and the scattering likely arise inside the Vela SNR itself where the line of sight intersects a dense clump of ionised material.

Expressed as a continuum flux density, the largest giant pulses have $1.4 \mathrm{GHz}$ flux densities at a distance of $1 \mathrm{kpc}$ of 4000, 200, 7 and $10 \mathrm{Jy}$ for the Crab, PSRs PSR B0540-69, 1821-24 and 1937+21 respectively for roughly the same number of rotations of the pulsar 
$\left(\sim 10^{6}\right)$. The giant pulses from the two millisecond pulsars have very similar flux densities and these are significantly less than those of the young pulsars. From the point of view of searching for giant pulses, the peak flux is the important value. The giant pulses in the Crab pulsar and in PSR B1937+21 are known to have widths of order microseconds. Assuming all the giant pulses have durations of $\sim 1 \mu \mathrm{s}$, then the giant pulses have peak fluxes at $1.4 \mathrm{GHz}$ at a distance of $1 \mathrm{kpc}$ of 130, 11, 0.03 and 0.02 MJy for the Crab, PSRs PSR B0540-69, 182124 and $1937+21$ which makes the differences between the young pulsars and the millisecond pulsars even more striking. In principle, therefore, the Parkes telescope is capable of detecting giant pulses from young pulsars at distances of $\sim 1 \mathrm{Mpc}$, and millisecond pulsars out to $\sim 50 \mathrm{kpc}$. In practise, however, lack of knowledge of the DM and scatter broadening both serve to weaken these limits. The prospects for future generation radio telescopes are excellent however. Even if, in young pulsars, the giant pulses only occur over the first $\sim 2000 \mathrm{yr}$, one might expect to see $\sim 20$ per large galaxy and more in the nearby starburst galaxies. For millisecond pulsars, the faster objects which have high magnetic fields at the light cylinder, will produce giant pulses for a Hubble time. There therefore may be a large population of millisecond pulsars which produce giant pulses, enhancing the prospects for targetted searches of globular cluster in external galaxies. Detection of extra-galactic pulsars will yield the electron density content of the inter-galactic medium, important in understanding the nature of the dark matter (Maloney \& Bland-Hawthorn 2001).

\section{Summary}

We have detected giant pulses from the young pulsar PSR B0540-69 in the LMC and yet have failed to detect the integrated flux from the pulsar after $8 \mathrm{hr}$ observing. Four pulsars, including PSR B0540-69, are now known to have giant pulses and these four are in the top five when ranked by their magnetic field at the light cylinder. In the near future we plan to observe PSR B0540-69 at both lower and higher radio frequencies to determine the spectral index of the giant pulse emission and also to replicate the detection of the pulsar at $0.64 \mathrm{GHz}$ by M93. We also plan a simultaneous radio and X-ray observation to both obtain accurate phasing between the radio and X-ray and to look for giant pulses in the X-ray.

The Australia Telescope is funded by the Commonwealth of Australia for operation as a National Facility managed by the CSIRO. We thank F. Marshall for providing sample RXTE pulse arrival times. We thank A. Lyne and D. Nice for providing timing ephemerides for the Crab and PSR B1937+21. 


\section{REFERENCES}

Camilo, F. et al. 2002, ApJ, 571, L41

Caraveo, P. A., Bignami, G. F., Mereghetti, S., \& Mombelli, M. 1992, ApJ, 395, L103

Cognard, I., Shrauner, J. A., Taylor, J. H., \& Thorsett, S. E. 1996, ApJ, 457, L81

de Plaa, J., Kuiper, L., \& Hermsen, W. 2003, A\&A, 400, 1013

Hankins, T. H., Kern, J. S., Weatherall, J. C., \& Eilek, J. A., Nature, 422, 141

Johnston, S., \& Romani, R. 2002, MNRAS, 332, 109

Kinkhabwala, A., \& Thorsett, S. E. 2000, ApJ, 535, 365

Lundgren, S. C., Cordes, J. M., Ulmer, M., Matz, S. M., Lomatch, S., Foster, R. S., \& Hankins, T. 1995, ApJ, 453, 433

Maloney, P., \& Bland-Hawthorn, J. 2001, ApJ, 553, L129

Manchester, R. N., Mar, D., Lyne, A. G., Kaspi, V. M., \& Johnston, S. 1993, ApJ, 403, L29 (M93)

Manchester, R. N., Staveley-Smith, L., \& Kesteven, M. J. 1993, ApJ, 411, 756

McConnell, D., McCulloch, P. M., Hamilton, P. A., Ables, J. G., Hall, P. J., Jacka, C. E., \& Hunt, A. J. 1991, ApJ, 249, 654

McLaughlin, M. A., \& Cordes, J. M. 2003, ApJ, Submitted

Middleditch, J., \& Pennypacker, C. 1985, Nature, 313, 659

Moffett, D. A., \& Hankins, T. H., ApJ, 522, 1046

Romani, R., \& Johnston, S. 2001, ApJ, 557, L93

Seward, F. D., Harnden, F. R., \& Helfand, D. J. 1984, ApJ, 287, L19

Zhang, W., Marshall, F. E., Gotthelf, E. V., Middleditch, J., \& Wang, Q. D. 2001, ApJ, 554, L177 


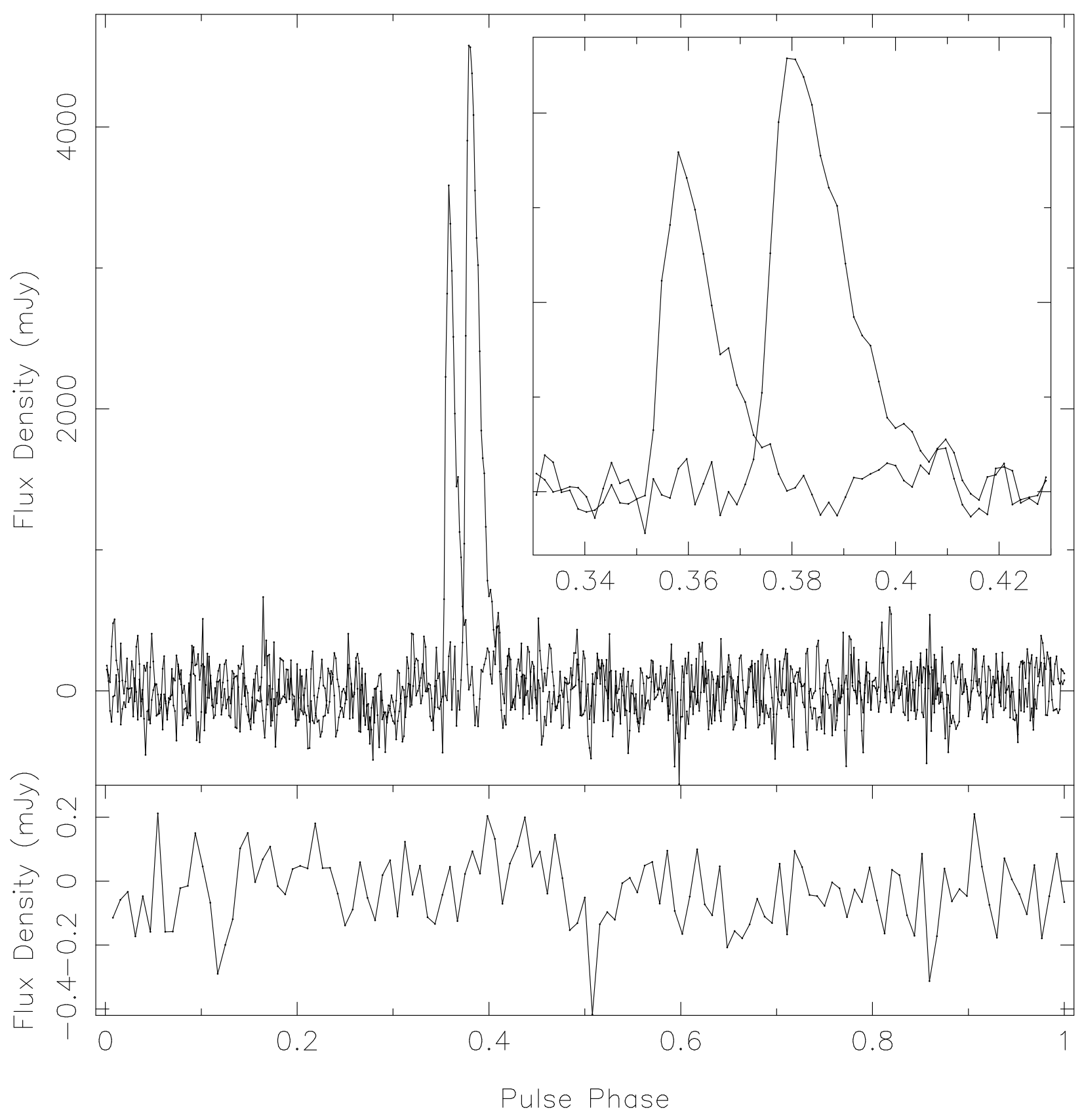

Fig. 1. - The bottom panel shows the profile of PSR B0540-69 at $1.4 \mathrm{GHz}$ after $8 \mathrm{hr}$ of integration. The full pulse period is shown subdivided into 128 phase bins. The flux density limit on pulsed emission with a $10 \%$ duty cycle is $13 \mu \mathrm{Jy}$. The top panel shows the two brightest giant pulses from PSR B0540-69 at the full resolution of $80 \mu$ s. The insert panel, with a restricted phase range, shows the sharp rise and exponential decay of the pulses. The peak of the X-ray emission occurs at phase 0.0 according to the ephermeris given in Section 2 although we caution there may be an unknown (significant) offset between the X-ray and radio timing. 


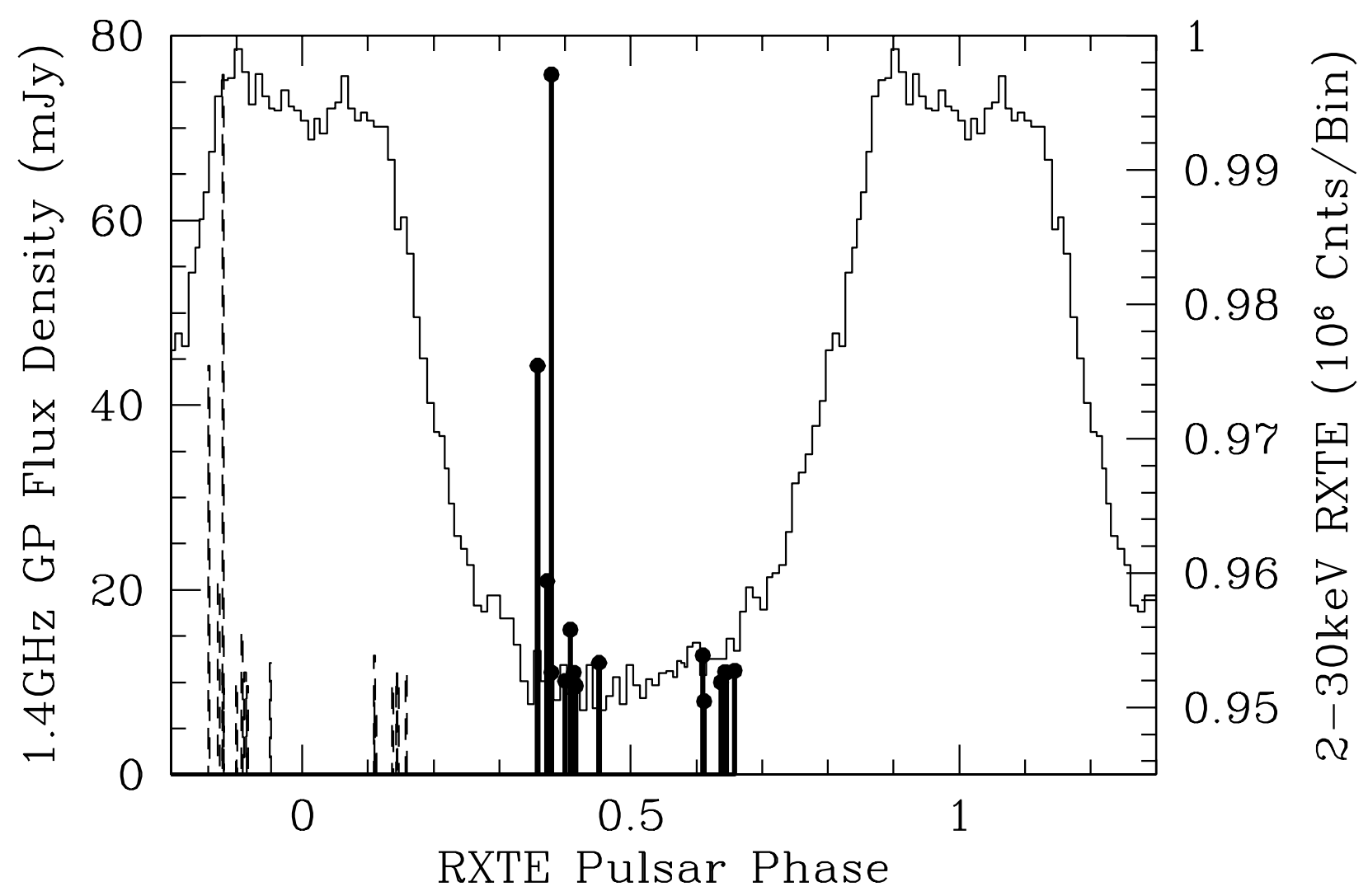

Fig. 2.- RXTE X-ray light curve (1.5 periods, after de Plaa et al 2003) with the nominal relative phases of the giant pulses shown as bold lines. Dashed lines give the giant pulses shifted in phase by 0.5 , showing the similarity between the spacing of the giant pulse phase windows and the peak structure in the high energy light curve. 


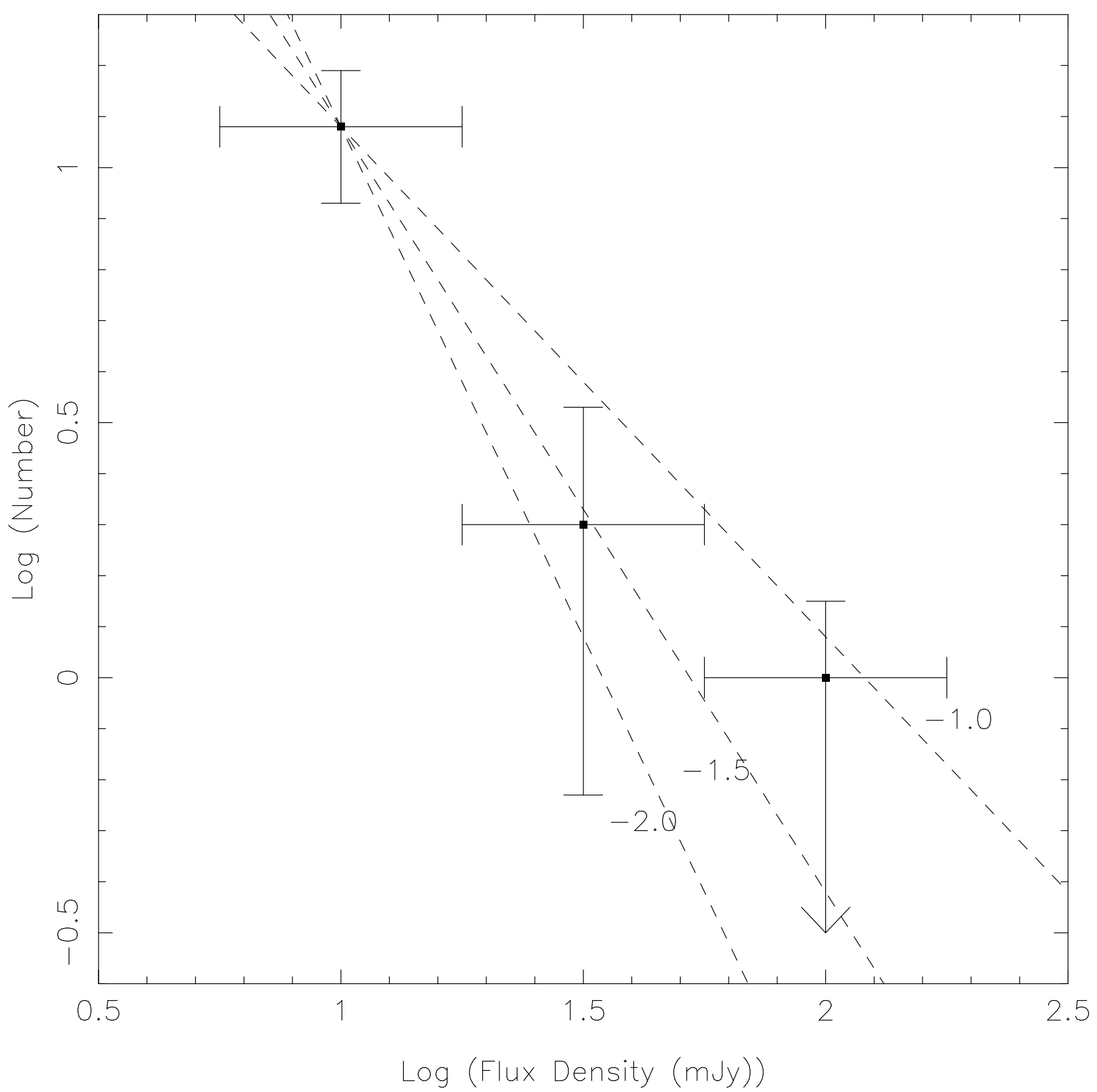

Fig. 3.- $-\log \mathrm{N}$ versus $\log \mathrm{S}$ for the 15 giant pulses along with sample slopes. The error bars in Log $\mathrm{N}$ simply reflect $\sqrt{N}$ statistics and those in Log $\mathrm{S}$ reflect the flux ranges used in the binning. 